\title{
Additional Comments Added to Our Recent Answer to G. Ghirardi
}

\author{
Elio Conte ${ }^{1,2}$ \\ ${ }^{1}$ School of Advanced International Studies on Applied Theoretical and Non Linear Methodologies of Physics, \\ Bari, Italy \\ ${ }^{2}$ Department of Basic Sciences, Neuroscience and Sense Organs, Univeristy Aldo Moro, Bari, Italy \\ Email: elio.conte@fastwebnet.it
}

Received 5 November 2014; accepted 18 November 2014; published 14 January 2015

Copyright (C) 2015 by author and Scientific Research Publishing Inc.

This work is licensed under the Creative Commons Attribution International License (CC BY). http://creativecommons.org/licenses/by/4.0/

(c) (i) Open Access

\section{Abstract}

Recently we published on International Journal of Theoretical Physics an answer to G. Ghirardi relating a paper published by this author in 1999. Since the argument is so complex and articulated, in the present paper we add further comments relating in particular the matter of the quantum collapse as we have elaborated it in our previous work using only Clifford algebra and thus reaching the basic result that it is a mechanism involving only information with a cybernetic collapse and retrocollapse dynamics as first it was introduced by Costa de Beauregard. We evidence as quantum collapse involves directly our psyche and our consciousness that of course is by itself an entity selecting alternatives on the basis of quantum rules. According to our previous experiments we also add comments on the possibility to estimate quantum interference at the level of perception and cognitive dynamics and reconstruct the wave function giving the possibility such result to discriminate between pure quantum superposition of states and mixtures just to re-confirm the role of quantum mechanics at the basic structure of mental entities.

\section{Keywords}

Quantum Collapse, Clifford Algebra, Information-Qubit, Quantum Cognition, Consciousness, Collapse-Retrocollapse of Wave Function

\section{New Comments}

Recently we published a paper on Int. Journal of Theoretical Physics (IJTP), entitled Answer to Giancarlo Ghirardi: Quantum Super Positions and Definite Perceptions: Envisaging New Feasible Experimental Tests. A Novel Proposal for Quantum Mechanics, Perception and Cognitive Science? [1], as answer to a previous paper 
[2] of this author that appeared time ago.

The purpose of this paper is to introduce further comments with respect to the published text of JITP in order to expose, to develop and to specify in more detail the issue.

The object of Ghirardi's paper is well clear and carefully understood. The paper was devoted to analyzing the possibility of checking experimentally whether the perceptual process can lead to the collapse of the wave function.

Apparently it could seem that in our response we left a lot of us from the object of Ghirardi issue but instead the connection is very clear if we look at some of our previous results. We intend to take into account the results that in 2010 we published on IJTP [3] under the title A Reformulation of von Neumann's Postulates on Quantum Measurement by Using Two Theorems in Clifford algebra and subsequently reconsidered in the framework of a Clifford reformulation of quantum mechanics in a paper that appeared in [4]. In the paper on IJTP for the first time we demonstrated that we can describe the quantum collapse using only the Clifford algebra and therefore only a rigorous mathematical basis. We are certainly aware that since the advent of quantum mechanics and in the following eighty seven years, the scientific literature has reported a considerable number of publications bearing physical models of interaction quantum system and measuring apparatus, but the peculiar and important aspect of our work is that we performed a demonstration using only algebra and proof of theorems without the aid of any topic of physics and in particular without the aid of a physical model of interaction microscopic quantum system-measuring apparatus or any kind of device. We demonstrated that the collapse of the wave function can be derived from the traditional Clifford algebra, that we call $A\left(S_{i}\right)$. When by our cognition we attribute a numerical value to one of the basic elements of the $A\left(S_{i}\right)$, we obtain a particular case of this algebra with new commutation rules. At the first inspection, it might seem that to attribute roughly a numeric value to one of the abstract elements of $A\left(S_{i}\right)$, is arbitrary, artificial, not linked to the process of quantum collapse. Instead, deepening the analysis, we demonstrated as a theorem that such a subalgebra, obtained by the assignment of a numerical value in $A\left(S_{i}\right)$, it is really a new Clifford algebra, $N_{+1}$, the dihedral algebra. It is generated every time by a direct cognitive attribution of a numerical value to a basic element of $A\left(S_{i}\right)$. By this way we had for the first time a mathematical proof of the collapse of the wave function. In the two papers, we discussed also in detail many cases of collapse that are of interest in traditional quantum mechanics and the analysis of each case confirmed the mathematical formulation introduced and the rigour of our development. There is another peculiar feature that we intend to outline here. The peculiar feature is also that we used only Clifford algebra. On the other hand we have reformulated the whole quantum mechanics by this algebra including also crucial features as EPR and Bell inequalities. The reason to use Clifford algebra is in an excellent paper of David Deutsch entitled It from Qubit [5] [6] (he recalls here the celebrated article of Wheeler It from Bit) where this author illuminates about the concept of information, on the basic problem existing in physics on "discrete or continuous", and concluding that the "world is made of qubits".

According also to Gottsemann and Chuang [7], Deutsch explained that we can describe a qubit in Heisenberg picture using the triple $q(t)=\left(q_{x}(t), q_{y}(t), q_{z}(t)\right)$ of Boolean observable $Q$ satisfying $q_{x}(t) q_{y}(t)=i q_{z}(t)$ and cyclic permutations over $(x, y, z)$ and $q_{x, y, z}^{2}(t)=1$. Everyone may acknowledge that these are just the basic elements of the $A\left(S_{i}\right)$ algebra that we used in our previously discussed mathematical demonstration of quantum collapse. Therefore, we cannot escape to conclude that we demonstrated that quantum collapse involves information. In detail a qubit is the fundamental unit of information, capable of existing in two states simultaneously or at a different time.

This result, obtained on the basis of strictly mathematical theorems and as devoid of any notion of physics and adoption of any physical model of interaction microscopic quantum system and measuring instrument, together with a number of other results obtained in recent years and strictly indicated in the bibliography of [1], have led us to the conclusion that the quantum collapse is a transition from a superimposed linear dynamics to a new dynamics in which a semantic act is involved. These two words require detailed explanation. What would we intend by semantic act. By semantic act we have to intend the direct involvement of information, relating mental entities whose consciousness is the basics representative. Never at any level we can talk about the collapse of the wave function if we do not introduce the semantic act and the wave function that, not in abstract but in concrete, is representative of the "factor of knowledge", the information, of mental entities involved. We have given also convincing results about the logical origins of quantum mechanics inverting the traditional and well known position of von Neumann [8] [9]. This is the basic reason because, answering to Ghirardi paper, we insisted on the argument of the superposition of states and we introduced convincing arguments about the quantum structure of 
mind entities and of consciousness, insisting on the manner in which mental entity and consciousness process information and articulate probabilities following the foundations of quantum mechanics. In these conditions a basic consideration arises. Everyone is of course free to give the scientific result of the importance that he believes and, in the particular case of quantum mechanics and the old debate about the nature and mechanism of the collapse of the wave function, each is perfectly free to continue to propose all the experiments retained of importance. What is meant to highlight here is that the results of the mathematics are another thing. The theorems are proved certainties. The ability to impact of reality by the mathematical tool should never be ignored.

There is another argument that it is necessary to deepen. As previously said, quantum collapse may be discussed only involving a semantic act and the role of our consciousness that selects on the basis of quantum rules. In addition, neurophysiology should be considered in this case: the visual system in humans and animals allows individuals to assimilate information from their surroundings. The act of seeing starts when the lens of the eye focuses an image of its surroundings into a light-sensitive membrane in the back of the eye, called the retina. Here is a crucial point: according to the standard view retina may be intended as a simple photodetector but really the retina is actually part of the brain, also if it is isolated to serve as a transducer for the conversion of patterns of light into neuronal signals. Again the relation to the role of quantum mechanics in the perceptual and cognitive processes is directly involved.

Finally, let us explain again what is the reason to consider quantum collapse and the importance to accept that it is induced by human mental entities. We evidenced previously that during quantum collapse transition the dynamics of information must be taken in serious consideration. As for the CT invariance of the Feynman propagator, the causality asymmetry it entails is factlike, not lawlike. The geometrical counterpart of the symmetry between prediction and retrodiction and between retarded and advanced waves, as expressed in the alternative expressions $\langle B \mid U A\rangle=\langle B U \mid A\rangle=\langle B|U| A\rangle$ for a transition amplitude between a preparation $|A\rangle$ and a measurement $|B\rangle$, is CPT invariant, not PT invariant. The previous three expressions respectively illustrate the collapse, the retrocollapse, and the symmetric collapse-retrocollapse concepts that were celebrated by Costa de Beauregard [10] with also some modest contributions by the author of this paper [11] [12]. Generally speaking, the transformation $|\psi\rangle=|U \phi\rangle$ or $|\phi\rangle=\left|U^{-1} \psi\right\rangle$ with $U$ evolution operator (of course given by us in [13] as member of the $A\left(S_{i}\right)$ Clifford algebra, exchanges preparation representation and measurement representation. Consequently, quantum collapse is not "attached by hand" to the fundamental deterministic evolution of a quantum system, as of course we demonstrated in [3], it corresponds to the cybernetic sequence coding-transmittingdecoding. Here we have the lawlike reversibility Negentropy $\Leftrightarrow$ Information, gain in knowledge, organizing power. The concept of information is a twofold entity: knowledge one could acquire, and an organizing power one could use. So the cyrcle of our discussion is closed. Quantum collapse is mathematically demonstrated by using Clifford algebra. Clifford algebra relates qubit that is the basic unit of information, ("the world is made of qubit”), quantum collapse involves information as gain in knowledge and organizing power: it is difficult to admit happening quantum collapse without the semantic act involving only information, thus our mental entities and the quantum manner in which consciousness operates.

This is the reason because quantum collapse is so important and it is a so complex action that cannot be ignored in every proposed experiment.

Finally we would add a final comment on other features introduced in the first answer. In fact, we have to remember here that in recent years we produced also a large number of experimental confirmations and in particular at the level of perception, using ambiguous figures but also at cognitive level using other basic processes of the neurobiology as the Stroop effect and some cognitive anomalies as the Conjunction fallacy. We gave evidence of violation of Bell's inequality at the cognitive level using the Clifford algebra. All the experiments confirmed that, in violation of the basic standard Bayes theorem, used probabilities confirmed the presence of the term of the quantum interference which is characteristic of only and only processes that fall under the domain of quantum mechanics. The estimate of the quantum interference factor is important not only to confirm the role of quantum mechanics in perceptive and cognitive level but because it allows us to reconstruct a posteriori the wave function of the system examined. It is well known that the wave function is not an observable entity in the formulation of quantum mechanics but can be rebuilt in retrospect, starting from the experimental estimate of the quantum interference factor. The confirmation of the presence of this abstract entity (the reconstructed wave function) at perceptual and cognitive levels is a marker evidencing the role of quantum mechanics at this level of our mental entities. In particular our formulation also allows discrimination between the quantum case instead of mixtures. This argument was processed in detail by us in many papers, and, in particular, in pages 530-531 of 
the paper Entitled "On the possibility that we think in a quantum probabilistic manner”, Neuroquantology [14]. In these pages it is evidenced the manner to distinguish between a quantum superposition linear dynamics and the case of a mixture.

\section{References}

[1] Conte, E. (2014) Answer to Giancarlo Ghirardi: Quantum Superpositions and Definite Perceptions: Envisaging New Feasible Experimental Tests. A Novel Proposal for Quantum Mechanics, Perception and Cognitive Science? International Journal of Theoretical Physics. http://dx.doi.org/10.1007/s10773-014-2259-6

[2] Ghirardi, G. (1999) Physics Letters A, 262, 1-14. http://dx.doi.org/10.1016/S0375-9601(99)00646-5

[3] Conte, E. (2010) International Journal of Theoretical Physics, 49, 587-614. http://dx.doi.org/10.1007/s10773-009-0239-z

[4] Conte E. (2011) Advanced Studies Theoretical Physics, 5, 485-544.

[5] Deutsch, D. (2012) It from Qubit. www.ipod.org.uk/reality/reality deutsch.pdf

[6] Conte, E. (2012) NeuroQuantology, 10, 561-565.

[7] Gottesman, D. and Chuang, I.L. (1999) Nature, 402, 390-393. http://dx.doi.org/10.1038/46503

[8] Conte, E. (2011) Electronic Journal of Theoretical Physics, 8, 109-126.

[9] Conte, E. (2011) NeuroQuantology, 9, 231-242.

[10] Costa de Beauregard, O. (1976) Foundations of Physics, 6, 539-559. http://dx.doi.org/10.1007/BF00715107

[11] Conte, E. (1981) Lettere al Nuovo Cimento, 32, 286-288. http://dx.doi.org/10.1007/BF02745121

[12] Conte, E. (1981) Lettere al Nuovo Cimento, 31, 380-382. http://dx.doi.org/10.1007/BF02776152

[13] Conte, E., Todarello, O., Federici, A., Vitiello, F., Lopane, M. and Khrennikov, A.Y. (2007) Chaos, Solitons and Fractals, 31, 1076-1088. http://dx.doi.org/10.1016/j.chaos.2005.09.061

[14] Conte, E. (2010) Neuroquantology, 8, 3-47. 
Scientific Research Publishing (SCIRP) is one of the largest Open Access journal publishers. It is currently publishing more than 200 open access, online, peer-reviewed journals covering a wide range of academic disciplines. SCIRP serves the worldwide academic communities and contributes to the progress and application of science with its publication.

Other selected journals from SCIRP are listed as below. Submit your manuscript to us via either submit@scirp.org or Online Submission Portal.
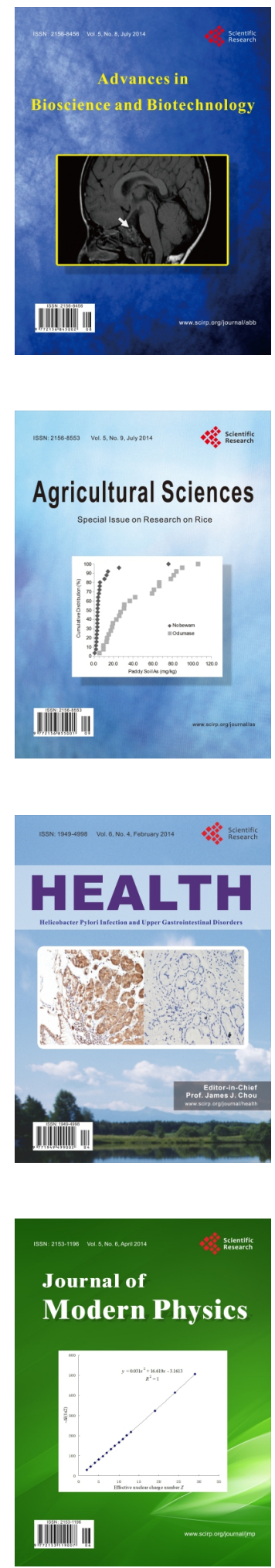
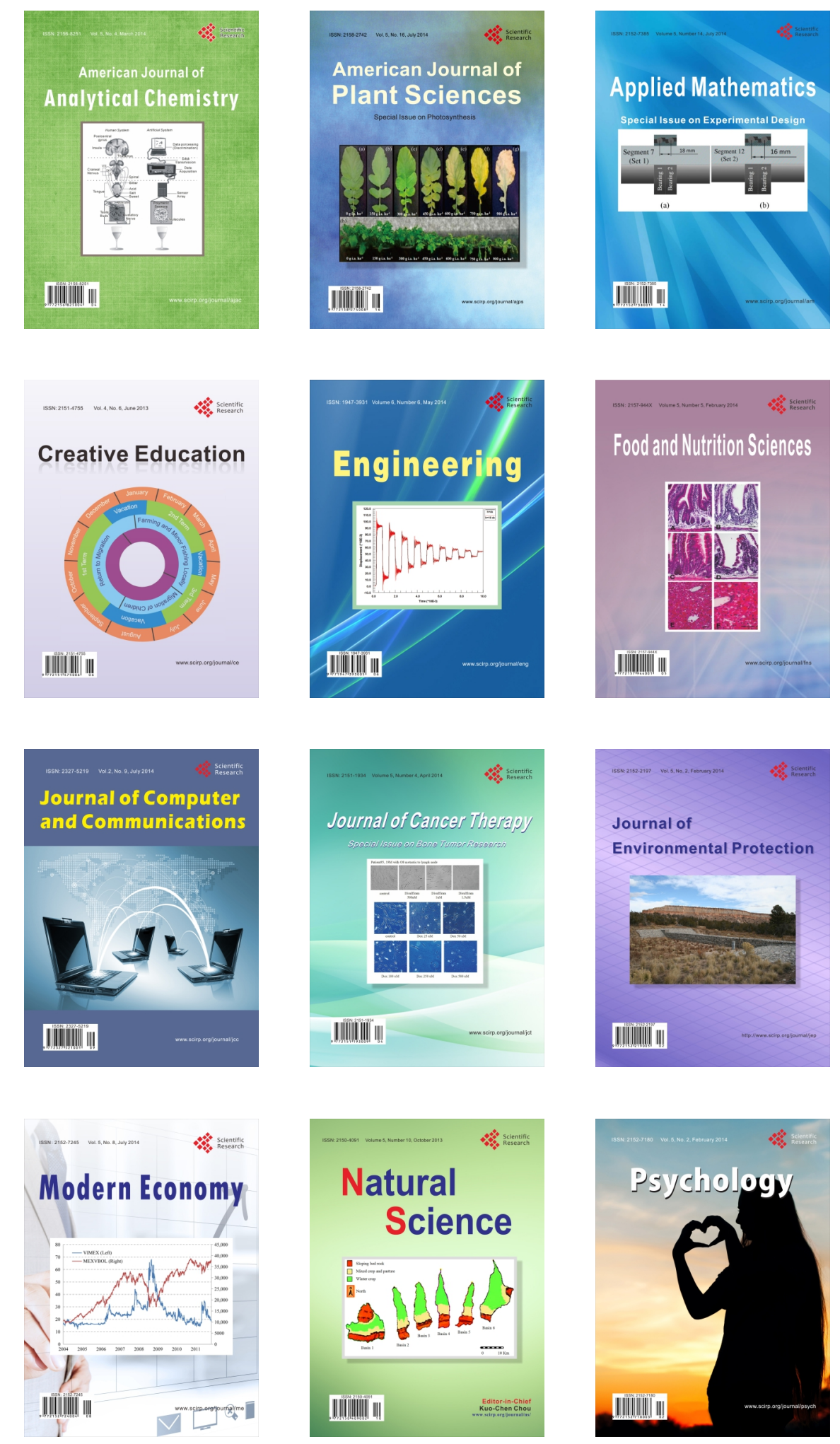